\title{
Peculiar behaviour of pure ENAW-AI99.5 sheet metal during uniaxial tensile test
}

\author{
Emil Yankov ${ }^{1, *}$, and Maria P. Nikolova ${ }^{1}$ \\ ${ }^{1}$ University of Ruse, Dept. Material Science and Technology, Ruse, 8 Studentska Str., Bulgaria
}

\begin{abstract}
Due to the generalized use of aluminium ( $\mathrm{Al})$ and $\mathrm{Al}$ alloy a detailed investigation of the mechanical and technological behaviour of a wide range of sheet metals is particularly needed. Often during deformation the $\mathrm{Al}$ sheets fold or crack which makes their subsequent use harder. The aim of this study is to identify the causes of the changes taking place during deformation that leads to earlier breaks of the Al sheet material. Pure ENAW-A199.5 in as-received condition is used for examination in order to determine its behaviour without interference of the alloying elements. After the uniaxial uniform tensile test significant differences in the mechanical strength of the samples cut out from the sheet material at three different directions $-0^{\circ}, 45^{\circ}$ and $90^{\circ}$ angle to the rolling direction are observed. The hardness values measured at three different directions: alongside the long side of the samples, cross-section and top view are compared. The XRD and microstructure analysis confirm the grain and lattice reorientation after the tests compared with the initial structure of the sheet material. These results could be applied as a basis of comparison when examining other $\mathrm{Al}$ alloys used in aviation and mechanical engineering, chemical and agro-food industry, etc.
\end{abstract}

\section{Introduction}

The wide application of aluminium $(\mathrm{Al})$ and its alloys in the modern mechanical engineering, car, aircraft, agro-food industry, etc., imposes a detail examination of the material behaviour during deformation and form-changing. For the purpose of improving its mechanical properties and plasticity $\mathrm{Al}$ is often alloyed with $\mathrm{Mn}$ and $\mathrm{Mg}$ and the materials thereof are used for higher levels of deep drawing. The behaviour of these alloys has been investigated by many authors [1-9] in order to determine the technological, mechanical, thermal, electro-chemical, etc. properties.

Over the years there have been signs that the pure $\mathrm{Al}$ is attracting less interest likely because of the low mechanical properties of the metal. In order to make a correct comparative analysis for the material behaviour, it could therefore be better the properties of the pure Al to be well defined. These properties afterward could be used as points of reference for future examination concerning the influence of the alloying elements during different types of testing.

\footnotetext{
*Corresponding author: eyankov@uni-ruse.bg
} 
The uniaxial uniform tensile test of technically pure $\mathrm{Al}$ sheet material cut at three different directions $-0^{\circ}, 45^{\circ}$ and $90^{\circ}$ in respect to the rolling direction is being subject to this investigation. The aim is to establish the mechanical properties in these three different directions and the reorientation of the crystallographic planes by means of XRD analysis. Additional evidence for the strengthening of the material is the change in the hardness values in three different directions of the tested samples.

\section{Experimental Procedures}

The material ENAW-A199.5 (ENAW-1050A) was received in the form of sheets with the following dimensions: $1000 \times 2000 \mathrm{~mm}$ and thickness of $\mathrm{t}_{0}=1.5 \mathrm{~mm}$ in annealed condition. Its chemical composition shown in Table 1 was measured by glow discharge optical emission analysis (GDOES), operated at $20 \mathrm{~mA}, 800 \mathrm{~V}$ and working pressure of $2.6 \mathrm{hPa}$.

Table 1. Chemical composition of ENAW-A199.5 (ENAW-1050A).

\begin{tabular}{|c|c|c|c|c|c|c|c|}
\hline Element & $\mathbf{F e}$ & $\mathbf{M n}$ & $\mathbf{T i}$ & $\mathbf{C u}$ & $\mathbf{M g}$ & $\mathbf{T i}$ & $\mathbf{A l}$ \\
\hline Wt.\% & 0.00 & 0.021 & 0.011 & 0.079 & 0.0089 & 0.0042 & Bal. \\
\hline
\end{tabular}

Table 2. Mechanical properties of ENAW-A199.5 (ENAW-1050A) at $20^{\circ} \mathrm{C}$.

\begin{tabular}{|c|c|c|c|c|c|}
\hline \multirow{2}{*}{$\begin{array}{c}\text { Mechanical } \\
\text { properties }\end{array}$} & \multirow{2}{*}{$\mathrm{R}_{\mathrm{m}}(\mathrm{MPa})$} & \multicolumn{3}{|c|}{ Elongation, A (\%) } & \multirow{2}{*}{ HV0.5 } \\
\cline { 3 - 5 } & $\mathrm{A}_{5}$ & $\begin{array}{c}\text { On a basis of } \\
\mathrm{L}_{0}=50 \mathrm{~mm}\end{array}$ & $\begin{array}{c}\text { On a basis of } \\
\mathrm{L}_{0}=65 \mathrm{~mm}\end{array}$ & \\
\hline Value & $74 \div 77.9$ & $24 \div 50$ & $18.9 \div 24.8$ & $27.6 \div 37.8$ & 24.7 \\
\hline
\end{tabular}

The mechanical properties (Table 2) of the as-received material were examined on a universal testing machine Instron-3384, on the base of standard ISO 6892-1:2009. A Vickers Hardness tester 432 SVD by Wilson-Wilpert was used for the hardness measurements of the metal according to the standard ISO 6507-1.

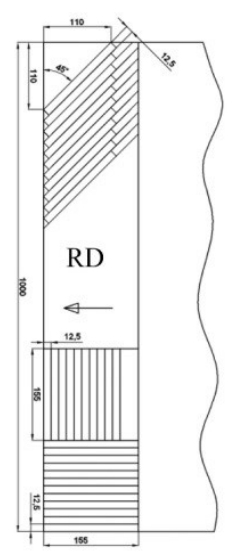

a)

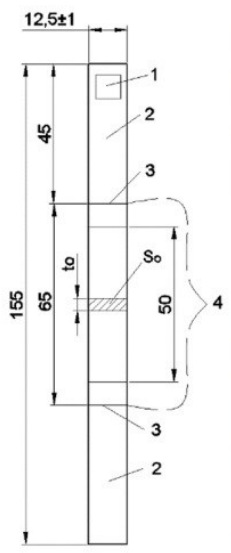

b)

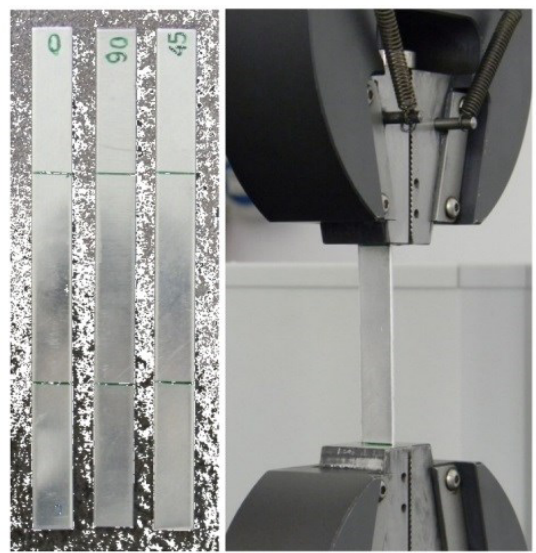

c) d)

Fig. 1. Sample preparation and testing: a) a scheme of cutting of the samples; b) shape and dimensions of the samples: 1) marking place; 2) gripping area of the clamps; 3) restraints; 4) tested area; $t_{0}$ - initial thickness, $\mathrm{S}_{0}$ - initial cross-sectional area; c) overall appearance of the samples; d) a fixed sample in the clamps of the machine. 
Samples were cut out from the sheet material at three different directions $-0^{\circ}, 45^{\circ}$ and $90^{\circ}$ to the RD as shown in Fig. 1a. The specimens used for the tensile test were sawn on the base of standard ISO 6892-1:2009 (Fig. 1a, b, c) and cut from the sheet by a guillotine with digital laser positioning. The material was tested in as-received (called undeformed) condition at ambient temperature. The static uniaxial tensile test was performed on a universal testing machine Instron-3384 (Fig. 1d) on the base of the same standard, supplied with a software Bluehill-3. For each test a control measurement of the working length of testing, height and width was held and the values were set in the used test method via the software program. For the purpose of the testing method a constant strain rate of $5 \mathrm{~mm} / \mathrm{min}$ until the load fell down to $100 \mathrm{~N}$ (or downturn of the maximum load below 90\%) was used. The data were saved in an appropriate digital form for subsequent analysis.

The surface preparation for hardness measurements of all samples before and after the tensile test (TT) involved grinding with grit paper (from P400 to P2500) and mechanical polishing with non-abrasive paste. For the hardness measurements three mutually perpendicular planes from each specimens cut near the break down zone were prepared. A Vickers Hardness tester 432 SVD by Wilson-Wilpert was used for the hardness measurements of the metal according to the standard ISO 6507-1. In order to compare the hardness changes the researches were made on three different planes: 1) alongside the long side of the sample (plane I); 2) cross-section (plane II) and 3) top view (plane III) of each (undeformed and TT) sample with a load of $0.5 \mathrm{~kg}$ and dwell time of $10 \mathrm{~s}$. Ten sequential measurements by increments of $50 \mu \mathrm{m}$ between the imprints were made for each plane.

The X-ray phase identification was performed with URD-6 diffractometer, applying Bragg-Brentano geometry and $\mathrm{Cu}-\mathrm{K}_{\alpha}$ radiation in the scanning range of $30-120^{\circ} 2 \theta$ with a step size of $0.05^{\circ} 2 \theta$ and counting time of $2.5 \mathrm{~s} / \mathrm{step}$. The $\mathrm{Cu}-\mathrm{K}_{\alpha}$ tube voltage and current were $30 \mathrm{kV}$ and $20 \mathrm{~mA}$, respectively. The lattice strain response was measured in the longitudinal and transverse directions of plane III of all samples. The qualitative phase analysis was held using Match!3 software. The texture analysis was performed by calculating the texture coefficient (TC) by the equation shown in [10].

\section{Experimental Results}

The representative indicator diagrams (Fig. 2a) are result of five samples testing from one the same angle of cutting sample $\left(0^{\circ}, 45^{\circ}\right.$ and $90^{\circ}$, respectively). The experimentally determined data indicate the load at maximal charge $F_{m}$ for tensile extension at maximum charge $\Delta \mathrm{L}_{\mathrm{m}}$, elongation at break $\Delta \mathrm{L}_{\mathrm{br}}$, elongation at tensile strength at allongement $\mathrm{A}$ and tensile strength $\mathrm{R}_{\mathrm{m}}$. The diagram considered as representative is the one with the closest mechanical properties to the average results from each indicator diagram for a particular angle of cutting [11].

As shown in Fig. 2a, both curves of $0^{\circ}$ and $45^{\circ}$ angle of cutting samples practically coincide, except for the last stage of deformation where the degree of relative elongation differs by more than $2 \mathrm{~mm}$. The mechanical strength of the $90^{\circ}$ cut samples is lower than the others but the change in the trajectory is like that of the other two directions of cutting. These phenomena can be attributed to the internal trans-crystal displacement, favourable lattice reorientation, grain fragmentation and other crystal effects.

A quantitative comparison of the mechanical properties of the material according to the tensile strength - tensile extension diagram is not possible because the real changes in the transverse section width have not been taken into account. Therefore, the $F=f_{1}(\Delta L)$ diagram (Fig. 2a) is converted into true stress - strain diagram $\sigma=f_{2}(\varphi)$, Fig. 2b, that takes account of equatorial section changes at any time compared to the tensile stress applied. The diagram indicates similar trend for the stress changes of the $0^{\circ}$ and $45^{\circ}$ angle of cutting samples and their mechanical strength values are slightly higher than those of $90^{\circ}$ cut 
samples. The true strain values do not exceed $41 \%$ which has been highlighted in previous examinations $[3,12]$. The true stress of the material ranges from 110 up to $118 \mathrm{MPa}$.

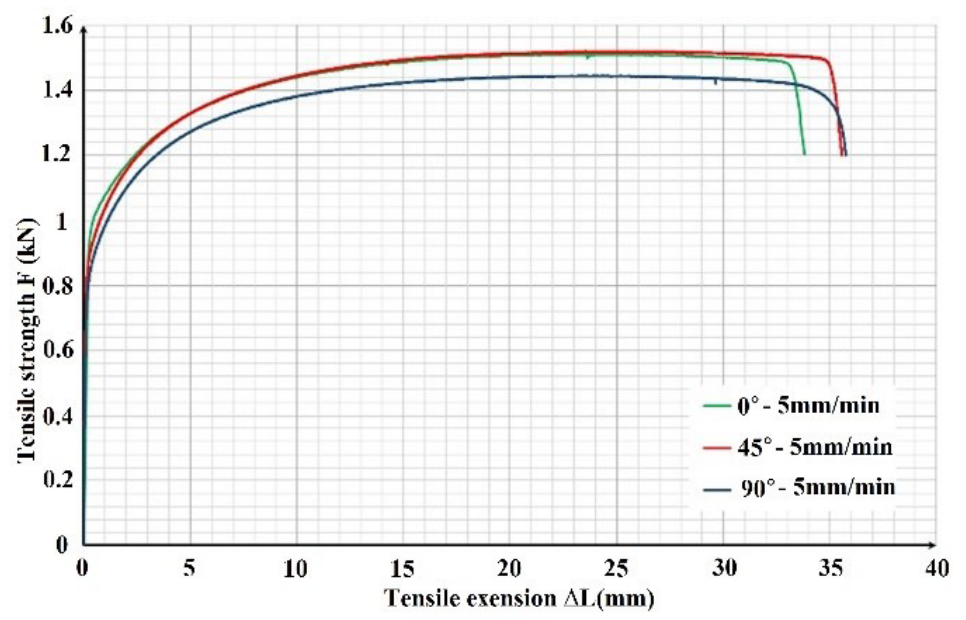

a)

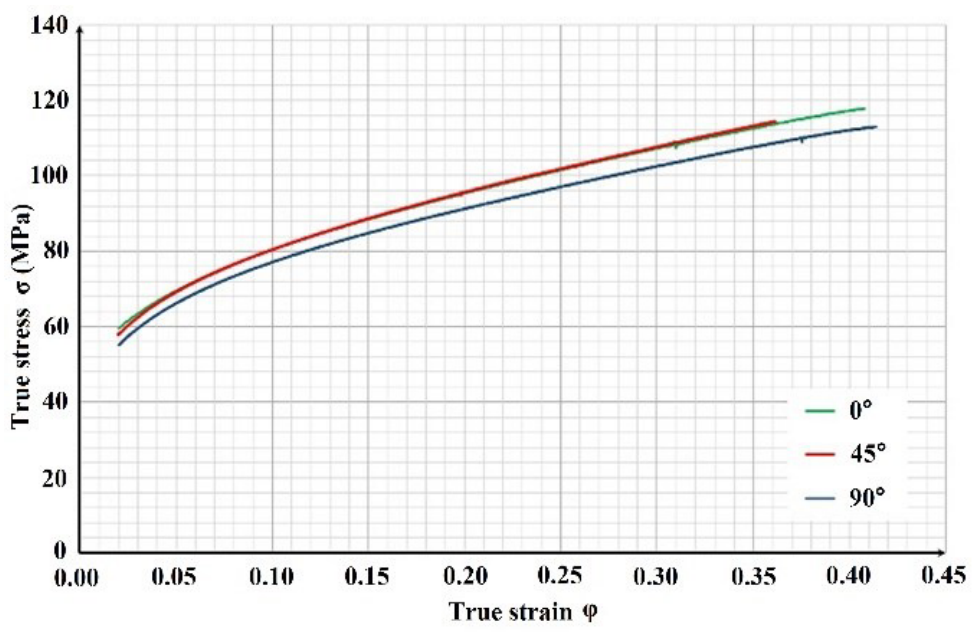

b)

Fig. 2. Representative indicator diagrams of ENAW-A199,5; $\mathrm{t}_{0}=1.5 \mathrm{~mm}$ : a) tensile strength - tensile extension, b) true stress - true strain.

To clarify the reasons for the differences in the mechanical strength, Vickers hardness measurements in orthogonal to each other planes and XRD measurements for analysis of the reorientation of the crystallographic planes could be used. The results of the average hardness values of planes I, II and III of the undeformed and TT samples cut at $0^{\circ}, 45^{\circ}$ and $90^{\circ}$ angle to the $\mathrm{RD}$ are listed in Excel tables and graphically shown in Fig. 3. Ten measurements are averaged for each plane (I, II and III) of a tested specimen. As the number of the directions examined is six, the total number of the completed tables is equal to eighteen. 


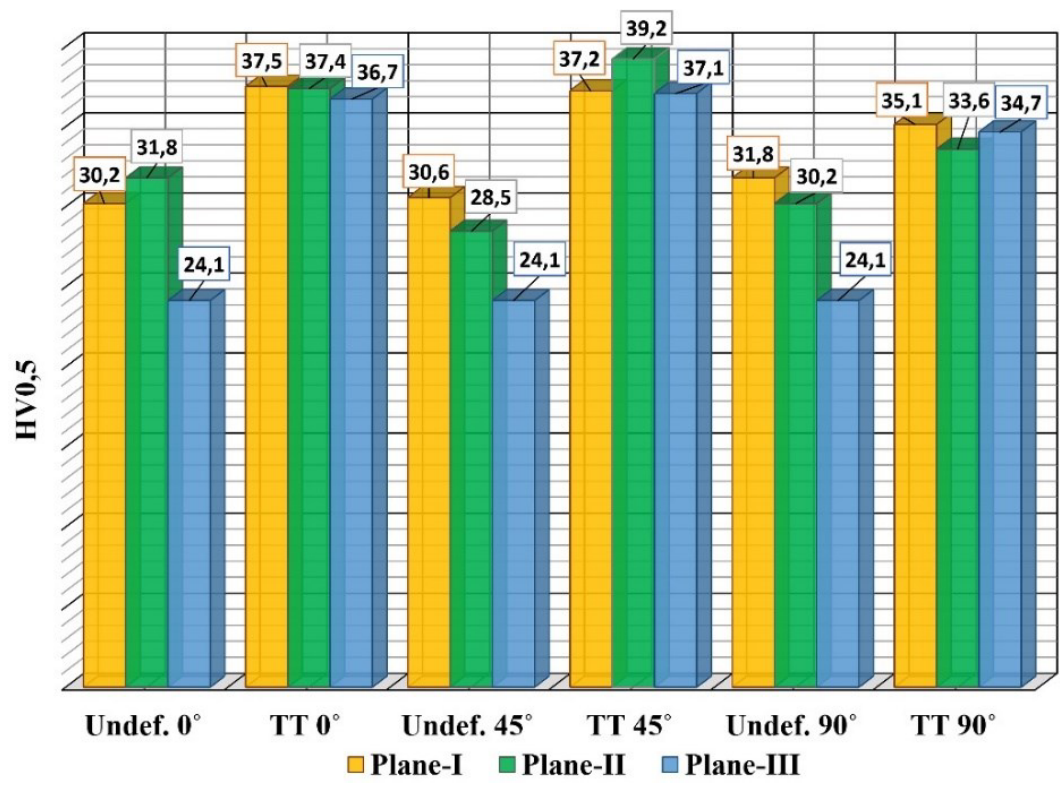

Fig. 3. Average hardness values before and after the tensile test of $0^{\circ}, 45^{\circ}$ and $90^{\circ}$ cut samples measured at planes I, II and III.

In as-received condition of the $0^{\circ}$ cut samples the lowest hardness values are measured in plane III and the highest - in plane II (Fig. 3). This difference is probably due to the initial pre-treatment - annealing of the undeformed metal that leads to surface softening while the sheet core remains not fully recrystallized. The observed phenomena will affect the trans-crystal movements during the uniaxial test. Conformation of this hypothesis would give a metallographic examination in planes I, II and III of all as-received and TT samples. The undeformed samples cut at $45^{\circ}$ and $90^{\circ}$ also show the lowest hardness value at plane III, while the highest values are measured at plane I. The reason for the higher hardness in plane I is the strengthening that runs perpendicular to the plane I within the $90^{\circ}$ cut sample and the texture direction within the $45^{\circ}$ cut sample that could be confirmed by XRD analysis.

After the tensile test the $90^{\circ}$ cut sample demonstrates the lowest tensile strength values. This fact also affects the results of the hardness values after the test (Fig. 3) that are lower than the analogue values of the $0^{\circ}$ and $45^{\circ}$ cut samples. The highest are the hardness values measured at plane I of the TT $90^{\circ}$ cut sample and the lowest are those in the perpendicular direction (plane II). This shows that the material has been subject to a hindered trans-crystal movements, but nevertheless, the hardness is increased by $10.5 \%, 11.26 \%$ and $43.6 \%$ in plane I, II and III, respectively (Fig. 4). The result gained shows that the sheet metal hasn't been fully recrystallized and the deformation takes place mainly on the surface.

For the TT $0^{\circ}$ cut sample the highest hardness values are measured at plane I and the lowest - at plane III (Fig. 3). Calculating the hardness changes in respect to the values of the undeformed $0{ }^{\circ}$ cut planes (Fig. 4), the highest strengthening is observed in plane III $(52.1 \%)$ and the lowest - in plane II $(17.55 \%)$.

Despite the highest hardness values measured in plane I for the as-received $45^{\circ}$ cut sample, after the tensile test the highest is hardness measured at plane II (Fig. 3). In contrast, the highest is the strengthening in plane III $(53.62 \%)$ and the lowest - in plane I (21.83\%), Fig. 4. 


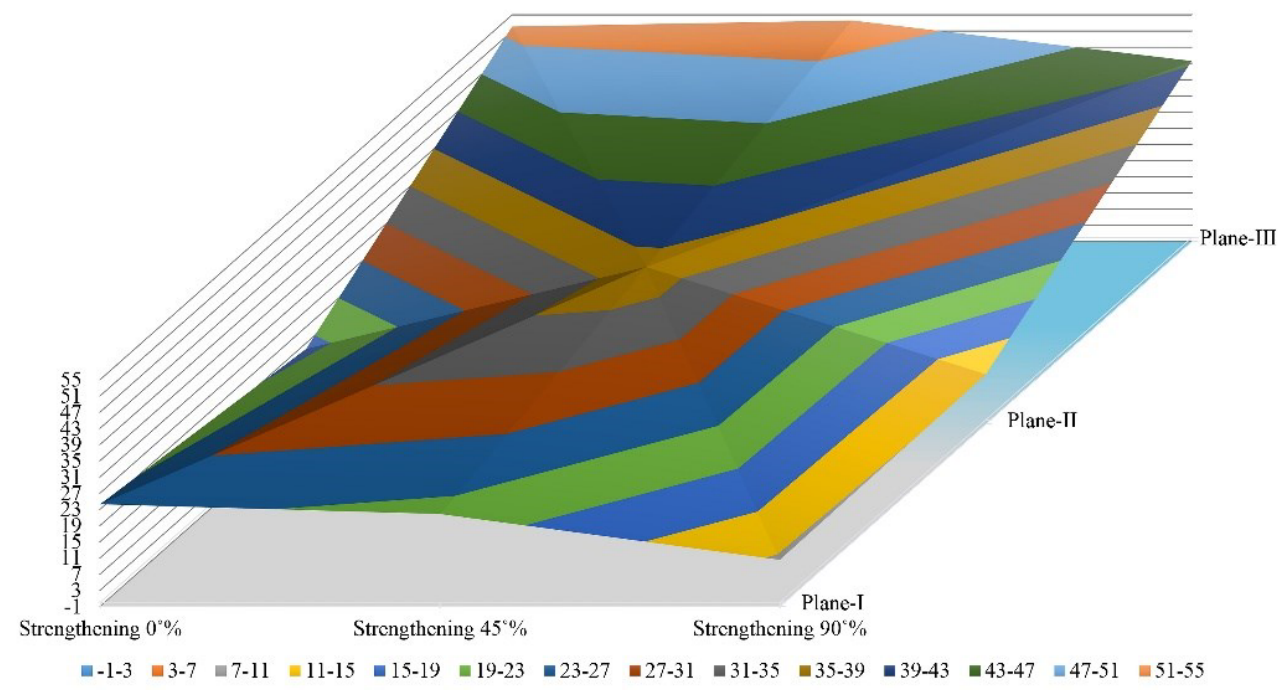

Fig. 4. Strengthening in percentage of the tensile tested samples $\left(0^{\circ}, 45^{\circ}\right.$ and $\left.90^{\circ}\right)$ applied to the undeformed samples for planes I, II and III.

The results thus obtained show that the highest is the strengthening in plane III for all three examined directions that is due to the softer sheet surface in as-received condition. For this reason, those planes of all samples are used for determining the changes in the crystallographic planes and texture of the material. The XRD analysis results obtained by a horizontal and vertical Bragg-Brentano scanning are summarized and shown in Fig. 5.

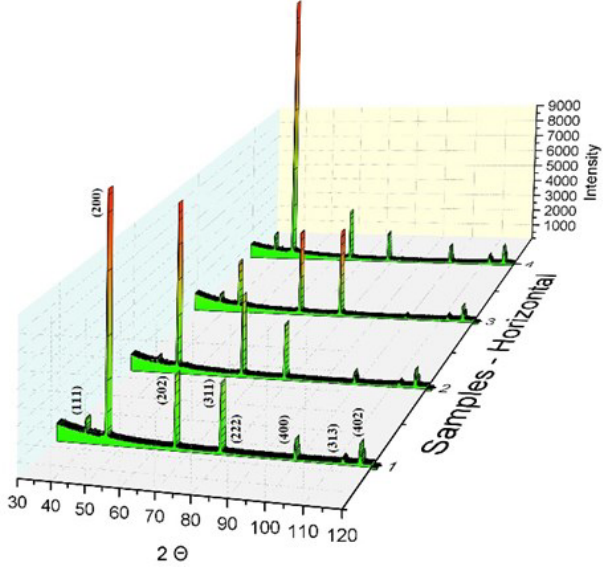

a)

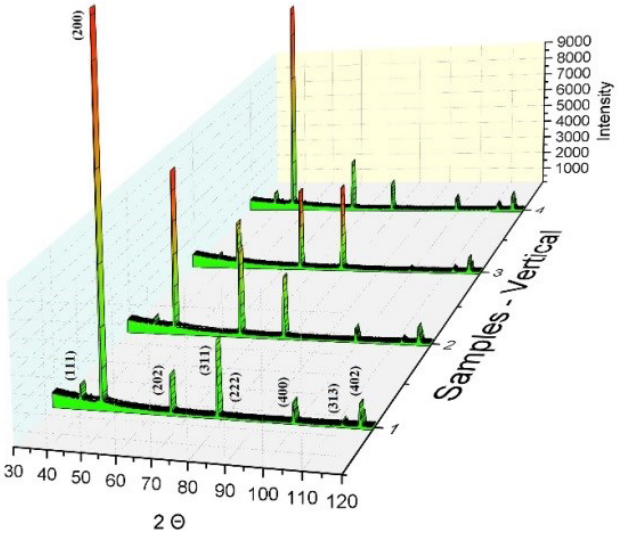

b)

Fig. 5. Summarized diffraction patterns of ENAW-A199.5 in initial state and after the tensile test of the samples in: a) horizontal direction and b) vertical direction of plane III. The numbered sample are as follows: 1 - undeformed; 2 - $0^{\circ}$ cut samples; 3 - $45^{\circ}$ cut samples; 4 - $90^{\circ}$ cut samples. The scanning geometry used corresponds to Bragg-Brentano (BB).

In as-received condition the highest is the intensity of the (200) plane both in horizontal and vertical directions, while the lowest is that of the (222) crystallographic plane (Fig. 5). According to the reference data the $100 \%$ reflection should be the (111) plane peak. This proves once more that there are residual traces of the initial deformation within the 
annealed metal. The inherited texture could explain the differences in the tensile strength values during the tensile test.

After the deformation of the $0^{\circ}$ cut sample scanned in horizontal direction the highest is still the intensity of (200) plane, because the grains do not undergo reorientation but elongation in the tensile direction. Nevertheless, the (200) intensity after the deformation decreases with $31 \%$ with respect to the undeformed peak intensity (Fig. 6a), possibly caused by grain refinement, reorientation or other crystallographic reasons. Reductions in the intensities of planes (111), (311), (400), (313) and (402) with 35.33, 17.03, 36.6, 18.04, $12.01 \%$, respectively are also observed for the account of an increase of (202) reflection intensity with $17.22 \%$. In the vertically scanned $0^{\circ}$ cut sample the (202) plane increases its intensity with $100.81 \%$ at the expense of the decrease of all the other planes intensities which is most relevant for (200) (53.17\%) and (400) (32.27\%) planes (Fig. 6b).

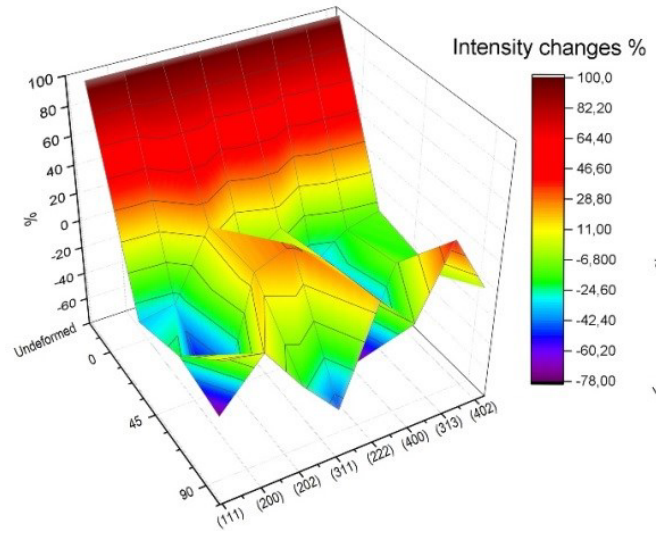

a)

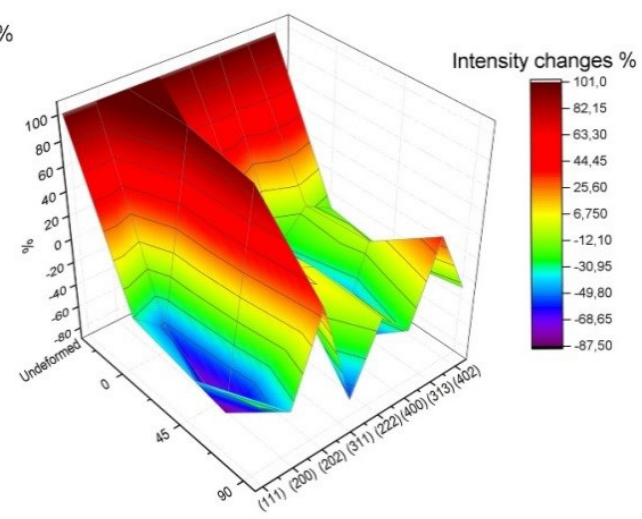

b)

Fig. 6. Intensity changes (in percentage) of TT $0^{\circ}, 45^{\circ}$ and $90^{\circ}$ applied to the underformed sample measured at planes III in: a) horizontal and b) vertical direction.

For the $45^{\circ}$ cut sample the highest are the intensities of (311) and (202) reflections that rise up to $30.32 \%$ and 19.75 , respectively in horizontal direction and 11.79 and $90.18 \%$, respectively, in vertical direction (Fig. 5). The rest of the crystallographic reflections decrease their intensities. Mainly affected are: (200) plane that reduces its intensity with $77.72 \%$ and $87.11 \%$ for the horizontal and vertical direction, respectively, and (400) plane with decreasing intensity of $76.09 \%$ and $77.79 \%$ for the horizontal and vertical direction, respectively (Fig. 6a, b). This significant difference in the reorientation of the crystals is due to the existence of favourable planes for trans-crystal movements applied to the direction of the tensile strength.

In the $90^{\circ}$ cut sample the intensity of (200) plane in horizontal and vertical direction of scanning is higher than that of the TT $0^{\circ}$ and $45^{\circ}$ cut samples (Fig. 5). Nevertheless, the (200) plane intensity decreases with $11.72 \%$ and $38.27 \%$ in horizontal and vertical direction, respectively, with respect to the undeformed sample. The highest is the reduction of (311) reflection intensity - with 50.04\% and 59.27\%, in horizontal and vertical direction, respectively. This decrease is for the account of the increase of (313), (222), (111), (200) and (402) plane intensities with $42.41 \%, 18.26,16.63,11.72,0.22 \%$, respectively in horizontal direction (Fig. 6a) and (313) and (202) with 37.67 and $31.91 \%$, respectively, in vertical direction (Fig. 6b). The strong reorientation of all planes in the horizontal direction is also caused by the direction of the applied tensile strength. Despite the unfavourable crystals' orientation, the (311) plane greatly reduces its intensity at the expense of (313) 
intensity increase. This reorientation is the only one possible, which accounts for the residual plasticity of the material cut at $90^{\circ}$ angle to the rolling direction and tensile tested.

The results obtained from the XRD analysis of plane III scanned in horizontal and vertical direction indicate clearly the favourable crystallographic changes occurring during the tensile test. Those planes that increase their intensities have been less deformed and textured for the account of the highly deformed and textured ones. The latter have higher initial intensities that subsequently decrease.

In order to confirm the results the texture coefficients (TC) for all reflections are calculated compared to the standard integrated intensity and shown in Fig. 7. The undeformed material is strongly textured in (400) and (200) and less textured in (202) and (311) planes both in horizontal and vertical directions. After the tensile test the (400) and (200) planes of $0^{\circ}$ and $90^{\circ}$ cut samples remain the strongest textured ones, but they slightly decrease mainly as a result of the increase of the TCs of (202), (313) and (402) for the $0^{\circ}$ cut samples and (202) and (313) for $90^{\circ}$ cut samples scanned in vertical direction while the other coefficients of the planes slightly amend.

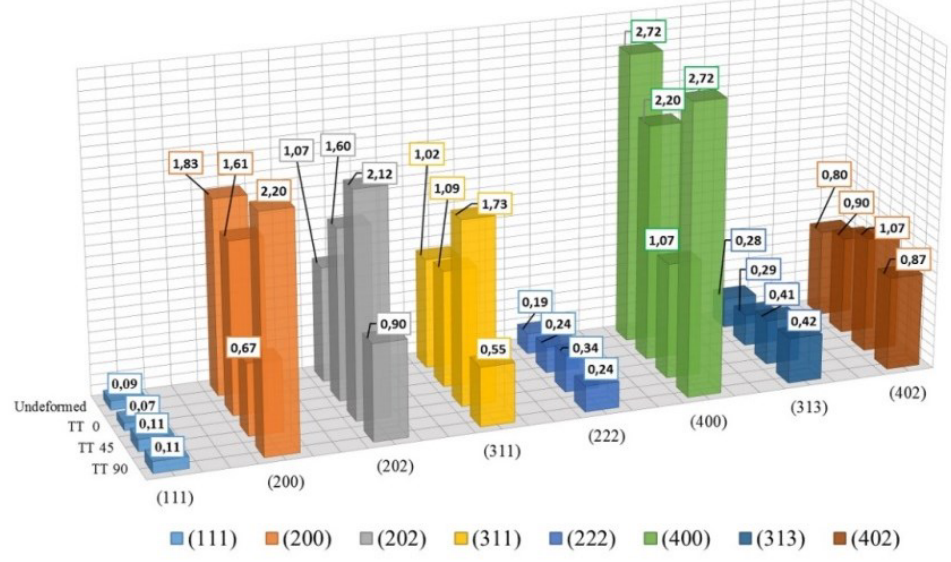

a)

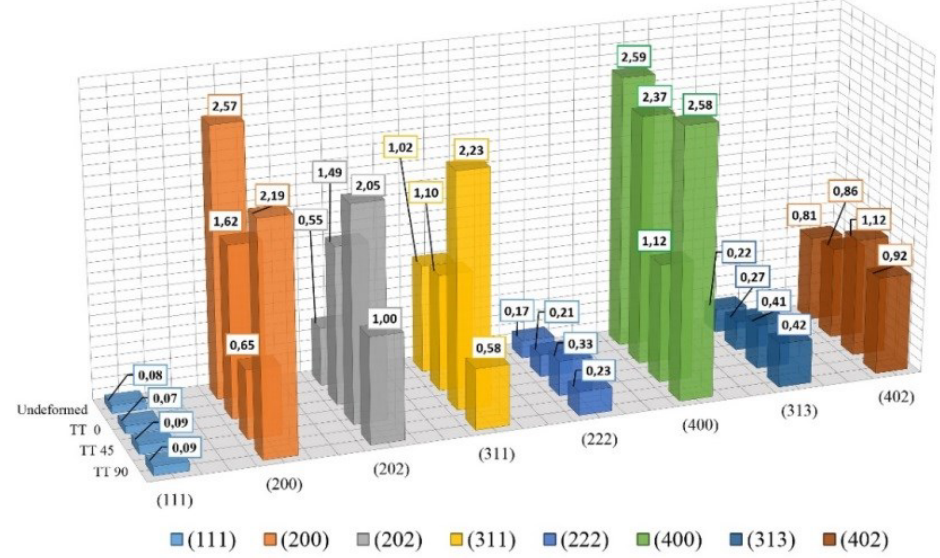

b)

Fig. 7. Calculated texture coefficients of the crystallographic planes of the undeformed and TT samples measured at planes III in: a) horizontal direction; b) vertical direction. 
In horizontal and vertical direction of the TT $45^{\circ}$ cut sample the strongly textured (400) and (200) planes greatly diminish their TCs for the account of changes of the TCs of the other planes. The strongest textured planes in horizontal direction are (202), (311), (402), (313) (222) and (111), while in vertical direction these are (311), (202), (402), (313) (222) and (111) planes.

The result of the calculated TCs reiterates the conclusion that the strongest changes occur in the crystallographic planes of the TT $45^{\circ}$ cut sample. The tensile test curves of the $0^{\circ}$ and $45^{\circ}$ cut sample merely overlap despite the different nature of the crystallographic and texture changes. It therefore follows that the most favorable trans-crystal movements are in planes (202) and (311), because both planes strongly change their TCs applied to the basic values of the undeformed sample.

Metallographic observations in each plane (I, II and III) of each undeformed and tested specimen with a subsequent software analysis for determining the grain sizes, orientations, distribution, etc., are further needed for fully explaining the mechanisms of strengthening, trans-crystal movement, texturing that will disclose thoroughly the peculiar behavior of ENAW-A199.5 sheet.

\section{Conclusions}

It has appeared from the various examinations that the annealed sheet material shows traces of residual texturing despite the heat treatment process that recrystallizes the surface material more than the sheet core. That's why the highest strengthening is observed in this softest surface zone - plane III for all three examined directions of cutting $-0^{\circ}, 45^{\circ}$ and $90^{\circ}$ angle to the rolling direction. The highest positive reorientation in both horizontal and vertical direction of scanning is seen in (202) plane in the case of all tested samples, with the exception of the $90^{\circ}$ cut sample scanned in horizontal direction where the TC decreases. The TC of the initially strongly textured (200) plane undergoes the strongest reduction after the tensile test of all samples scanned in horizontal and vertical direction, with the exception of the TT and horizontally scanned $90^{\circ}$ cut sample where the TC slightly increases. The calculated TC clarifies the mechanisms of the trans-crystal movements that follow a decrease in the values of the textured planes for the account of slightly textured or non-textured planes with stocks of residual plasticity. The latter increases their TCs enabling greater deformation.

Additional microstructural analysis after the uniaxial tensile test in planes I, II and III of the undeformed and TT samples cut at $0^{\circ}, 45^{\circ}$ and $90^{\circ}$ angle to the RD needs to be further fully exploited for clarification of the peculiar behaviour of the sheet material.

\section{References}

1. M. T. Di Giovanni, E. Cerri, D. Casari, M. Merlin, L. Arnberg, and G. Garagnani, Metall. Mater. Trans. A 47 (5), 2049 (2016)

2. A. Zykova, L. A. Kazantseva, I. A. Kurzina, V. Kh. Dammer, and A. V. Chumaevskii, Tsvetn. Met. (Moscow), 5, 4 (2015)

3. T. A. Kalashnikova, S. Tarasov, A. V. Chumaevskii, F. Andrey, E. A. Kolubaev, V. E. Rubtsov, A. Eliseev, and V. A. Beloborodov, Advanced Materials with Hierarchical Structure for New Technologies and Reliable Structures, AIP Conf. Proc., 020079 (2016)

4. M. Akbarifar, M. Divandari, Conference: Iran International Aluminum Conference (IIAC2014), (2014)

5. X. Wu , N. Tao, Y. Hong, B. Xu , J. Lu , K. Lu, Acta Mater., 50, 2075 (2002)

6. K. Dai, J. Villegas, Z. Stone, L. Shaw, Acta Mater., 52 (2), 5771 (2004)

7. R. Kapoor, J.B. Singh, J.K. Chakravartty, Mater. Sci. Eng. A, 496, 308 (2008) 
8. R.Z. Valiev, N.A. Enikeev, M.Yu. Murashkin, V.U. Kazykhanov, X. Sauvage, Scr. Mater., 63 (9), 949 (2010)

9. P. H. R. Pereira, Y. C. Wang, Yi Huang, T. G. Langdon, Mater. Sci. Eng. A, 685, 367 (2017)

10. E. Yankov, M. Nikolova, MATEC web of Conferences, CoSME'16, 94, (2017)

11. E. Yankov, Sheet materials testing using biaxial hydraulic bulge test, Dissertation, Ruse, 112 (2014)

12. S. Dumoulin, O. Engler, O. S. Hopperstad and O.-G. Lademo, Modelling and Simulation in Materials Science and Engineering 20 (5), 1-20 